\title{
Analysis of the Full Isoparametric Multigrid Algorithm for a Second Order Elliptic Problem
}

\author{
Todor D. Todorov*
}

Technical University, 5300 Gabrovo, Bulgaria

\begin{abstract}
A second order elliptic problem is investigated in domains with complex geometry. The sequence of nonnested finite element triangulations is generated by higher order curved elements. Convergence analysis of the full nonnested multigrid algorithm is done on the basis of a pure isoparametric approach. A numerical example supporting the considered theory is presented.
\end{abstract}

Keywords: Nonnested multigrid method, isoparametric approach, lipschitz-continuous boundary.

\section{INTRODUCTION}

The reaction-diffusion equation in curved domains is an object of interest in our investigation. Most of the authors considering such problems analyze various methods on the basis of piecewise linear finite elements [1-7]. Such elements are applicable in the case of polygonal domains with simple geometry. Curved domains with complex geometry needs higher order elements and isoparametric or superparametric approach [8]. On the other hand multigrid method is the most powerful tool for solving finite element equations.

There are various reasons to obtain non-nested finite element spaces when solving elliptic boundary value problems. Mixed finite element formulations, some $C^{1}$ finite elements [4], non-quasi-uniform [6] or degenerate [7] triangulations, noninherited bilinear forms [1] tend to generate non-nested multigrid methods. The application of finite element methods for solving problems in curved domains requires isoparametric approach. Usually isoparametric finite element spaces are non-nested [9].

M. Jung and T. Todorov considered a two-level method for the problem of interest and proved the stability of a intergrid transfer operator [9]. Such results are a necessary but not a sufficient condition for convergence of a multigrid method. Moreover various multigrid methods can be constructed using the same two-level method. In order to conclude convergence for the full multigrid algorithm in the nested case the above could meet aptly the requirement stated by means of employing recursion properties. But in the case of curved boundaries convergence of the full multigrid algorithm depends on the properties of the isoparametric finite element spaces.

In the present paper multigrid extension of the two-level method obtained by M. Jung and T. Todorov [9] is inves-

\footnotetext{
*Address correspondence to this author at the Technical University, 5300 Gabrovo, Bulgaria; Tel: 0035966 873016;

E-mail: t.todorov@yahoo.com
}

tigated and convergence results are found. A rigorous proof of optimal rate of convergence for the full isoparametric non-nested multigrid algorithm is done. The analysis is completed for all degree of trial functions greater than one. Asymptotic rate of convergence is computed numerically thereby confirming the main result.

\section{SETTING OF THE PROBLEM}

Let $\Omega$ be a simply connected bounded curved domain in $\mathbf{R}^{2}$, with Lipschitz-continuous boundary $\Gamma$. Consider the Dirichlet problem for the nonhomogeneous reactiondiffusion equation

$$
(P):\left\{\begin{array}{rlcc}
\text { Find a function } u \text { such that } & & & \\
-\nabla \cdot(a(x) \nabla u)+b(x) u & = & f & \text { in } \Omega, \\
u & = & 0 & \text { on } \Gamma
\end{array},\right.
$$

where the coefficients $a(x), b(x)$ and the right-hand side $f(x)$ are sufficiently smooth functions. Assume that there exist positive constants $\underline{a}, \bar{a}, \underline{b}$ and $\bar{b}$, such that

$$
\underline{a} \leq a(x) \leq \bar{a}, \underline{b} \leq b(x) \leq \bar{b}, \forall x \in \bar{\Omega} .
$$

As usual, we denote the real Sobolev space for nonnegative integers $k$ and $p=2$ by $H^{k}(\Omega)$. The space $H^{k}(\Omega)$ is provided with the norm $\|\cdot\|_{k, \Omega}$ and the seminorm $|\cdot|_{k, \Omega}$. Define the space

$$
H_{0}^{1}(\Omega)=\left\{v \in H^{1}(\Omega) \mid v=0 \text { on } \Gamma\right\} .
$$

The weak formulation of problem $(P)$ is given by

$$
(\mathcal{P}):\left\{\begin{array}{l}
\text { Find a function } u \in \mathbf{V}=H_{0}^{1}(\Omega) \text { that satisfies } \\
a(u, v)=(f, v), \quad \forall v \in \mathbf{V}
\end{array}\right.
$$


with

$a(u, v)=\int_{\Omega}\{a(x) \nabla u \cdot \nabla v+b(x) u v\} d x \quad$ and $\quad(f, v)=\int_{\Omega} f v d x$.

\section{ISOPARAMETRIC FINITE ELEMENT DISCRETI- ZATIONS}

Let $\tau_{0}$ be an initial triangulation of the domain $\Omega$ with triangular finite elements of degree $k \geq 2$. Normally, curved elements are used only in the boundary layer. The domain $\Omega_{0}$ corresponds to the initial triangulation. Dividing each element of $\tau_{n} \quad n \geq 0$ into $k^{2}$ finite elements of degree $k$ we obtain the triangulation $\tau_{n+1}$. The approximate domains $\Omega_{n}$ correspond to the triangulations $\tau_{n}$. Denote $\Gamma_{n}=\partial \Omega_{n}$. Obviously $\Omega_{n-1} \not \equiv \Omega_{n}$ and $\Omega_{n} \not \equiv \Omega$. Let $\mathcal{N}_{n}$ be the set of all nodes of the triangulation $\tau_{n}$ and $\tilde{\mathcal{N}}_{n}=\mathcal{N}_{n} \backslash \mathcal{N}_{n-1}$. The sets of the nodes of two consecutive triangulations are connected by $\mathcal{N}_{n-1} \subset \mathcal{N}_{n}$. The nodes in $\tilde{\mathcal{N}}_{n} \cap \Gamma$ could not belong to $\Gamma_{n-1}$. For more details see M. Jung and T. Todorov [9]. Define a finite element space $\mathbf{V}_{n}$ associated with a triangulation $\tau_{n}$ by

$$
\mathbf{V}_{n}=\left\{v \in C\left(\Omega_{n}\right) \mid v_{\mid K} \in P_{K}, K \in \tau_{n}\right\} .
$$

Let $\Phi_{n}: \Omega_{n} \rightarrow \Omega$ be the map defined by Lenoir [10]. The map $\Phi_{n}$ is notable with the fact that the distance from any point on $\Gamma$ to the closest point on $\Gamma_{n}$ is $O\left(h_{n}^{k+1}\right)$ at most when the boundary is smooth enough. Moreover $\Phi_{n}$ is equal to the identity map on elements, which do not belong to the boundary layer.

Introduce the approximating bilinear forms and the $L_{2}$ scalar product in $\mathbf{V}_{n}$ by

$$
\begin{gathered}
a_{n}(u, v)=\int_{\Omega_{n}}\left\{\left(a \circ \Phi_{n}\right)(x) \nabla u \cdot \nabla v+\left(b \circ \Phi_{n}\right)(x) u v\right\} d x, \forall u, v \in \mathbf{H}_{0}^{1}\left(\Omega_{n}\right), \\
(u, v)_{n}=\int_{\Omega_{n}} u v d x, \forall u, v \in \mathbf{V}_{n} .
\end{gathered}
$$

Now we are able to formulate the problem $\left(\mathcal{P}_{n}\right)$ approximating $(\mathcal{P})$

$\left(\mathcal{P}_{n}\right):\left\{\begin{array}{l}\text { Find a function } u_{n} \in \mathbf{V}_{n} \text { that satisfies } \\ a_{n}\left(u_{n}, v\right)=\left(f \circ \Phi_{n}, v\right)_{n} \stackrel{\text { def }}{=} G_{n}(v), \quad \forall v \in V_{n}\end{array}\right.$

\section{MULTILEVEL SOLUTION METHOD}

Consider the eigenpairs $\left(\lambda_{i}^{(n)}, \psi_{i}^{(n)}\right)$ of the problem

$a_{n}\left(\psi_{i}^{(n)}, v\right)=\lambda_{i}^{(n)}\left(\psi_{i}^{(n)}, v\right)_{n}, \forall v \in \mathbf{V}_{n}$,

$i=1,2, \ldots, N_{n}=\operatorname{dim} \mathbf{V}_{n}$.

Without loss of generality we suppose that the eigenfunctions are normalized by the following way $\left(\psi_{i}^{(n)}, \psi_{j}^{(n)}\right)_{n}=\delta_{i j}, a_{n}\left(\psi_{i}^{(n)}, \psi_{j}^{(n)}\right)=\lambda_{i}^{(n)} \delta_{i j}$, where $\delta_{i j}$ is the Kronecker symbol. For each $v$ in $\mathbf{V}_{n}$ we have the following representation $v=\sum_{i=1}^{N_{n}} c_{i} \psi_{i}^{(n)}$. Define the norm $\left|\|\cdot \mid\|_{s, n}\right.$ by

$\|v\|_{s, n}^{2}=\sum_{i=1}^{N_{n}} c_{i}^{2}\left(\lambda_{i}^{(n)}\right)^{s}, s \geq 0$.

Obviously,

$\|\cdot \mid\|_{0, n}=\|\cdot\|_{0, \Omega_{n}}$ and \|\|$\cdot\left\|_{1, n}=\right\| \cdot \mid\|\|_{n} \simeq\|\cdot\|_{1, \Omega_{n}}$,

where \|\|$\cdot \mid \|_{n}$ is the usual energy norm. Denote the intergrid transfer operator [9] by $I_{n}: \mathbf{V}_{n-1} \mapsto \mathbf{V}_{n}$. The auxiliary operator $Q_{n-1}: \mathbf{V}_{n} \mapsto \mathbf{V}_{n-1}$ introduced by $a_{n-1}\left(Q_{n-1} w, v\right)=a_{n}\left(w, I_{n} v\right), \forall v \in V_{n-1}, \forall w \in \mathbf{V}_{n}$

defines the coarse grid correction in the nonnested multigrid method. Compile the coarse-level residual problem

$\left(\mathcal{R}_{n-1}\right):\left\{\begin{array}{l}\text { Find a function } \bar{\varepsilon} \in \mathbf{V}_{n-1} \text { such that } \\ a_{n-1}(\bar{\varepsilon}, v)=G_{n}\left(I_{n} v\right)-a_{n}\left(w^{(v)}, I_{n} v\right) \quad \forall v \in \mathbf{V}_{n-1},\end{array}\right.$

for some $w^{(v)} \in \mathbf{V}_{n}$. Denote the full multigrid solution obtained by $r$ iterations of the $n$-th level scheme (2) with initial guess $I_{n} \hat{u}_{n-1}$ by $\hat{u}_{n}$.

The $n$-level algorithm $(n \geq 1)$.

For $n=0$, solve $\left(\mathcal{P}_{0}\right)$ by any method.

obtain $u_{0}$

set $\hat{u}_{0}=u_{0}$

For $n>0$ do the following

set $u^{[0]}=I_{n} \hat{u}_{n-1}$

For $i=1$ to $r$ do

set $w^{(0)}=u^{[i-1]}$

(i) perform $v$ smoothing iterations with the initial guess $w^{(0)}$

$\left(w^{(j)}-w^{(j-1)}, v\right)_{n}=\Lambda_{n}^{-1}\left(G_{n}(v)-a_{n}\left(w^{(j-1)}, v\right)\right), v \in \mathbf{V}_{n}$,

$j=1,2, \ldots, v$, where $\Lambda_{n}$ is the largest eigenvalue of the problem (1).

(ii) Solve the residual problem $\left(\mathcal{R}_{n-1}\right)$ approximately by means of $p$ iterations of the $(n-1)$ st-level scheme starting with the initial guess zero for obtaining an approximate solution $\varepsilon$.

(iii) Compute the new iterate $w^{(v+1)}$ by

$$
w^{(v+1)}=w^{(v)}+I_{n} \varepsilon .
$$

set $u^{[i]}=w^{(v+1)}$

next $i$

obtain $\hat{u}_{n}=u^{[r]}$

next $n$. 


\section{PRELIMINARY RESULTS}

Describe some hypotheses, which are necessary for the convergence analysis.

H1 The boundary $\Gamma$ is piecewise $C^{k+1}, k \geq 2$.

H2 All nodes from $\mathcal{N}_{n} \cap \Gamma_{n}$ belong to $\Gamma$.

H3 The triangulations $\tau_{n}$ consists of isoparametric finite elements of degree $k \geq 1$ in the boundary layer and straight elements in the rest of $\Omega_{n}$.

H4 The triangulations $\tau_{n}$ are $k$-regular in the sense of Ciarlet and Raviart [11]

H5 The weak solution $u \in H^{k+1}(\Omega)$.

Lemma 1 [9, M. Jung and T. Todorov]. Let hypotheses HI$H 4$ be fulfilled, then there exists a constant $\alpha \in[0,1)$ such that the inequality

$k-\alpha \leq \frac{h_{n-1}}{h_{n}} \leq k+\alpha, \forall k \geq 2, \forall n \in \mathbf{N}$

holds for any sufficiently small $h_{n-1}$.

The next theorem certifies the convergence of the twolevel method.

Theorem 1 [9, M. Jung and T. Todorov] Suppose that conditions H1-H5 are satisfied and $p>1$ be any integer.

Then there exist constants $v \geq 1$ and $\gamma \in(0,1)$ independent of the level number $n$, such that

$$
\|\| u_{n}-w^{(v+1)}\|\|_{n} \leq \gamma\left\|\mid u_{n}-w^{(0)}\right\|_{n}
$$

if

$$
\|\| \bar{\varepsilon}-\varepsilon\left\|_{n-1} \leq \gamma^{p}\right\| \bar{\varepsilon} \|_{n-1} .
$$

\section{CONVERGENCE OF THE FULL MULTIGRID ALGORITHM}

[9, Theorem 2] assures the stability of the intergrid transfer operator $I_{n}$. Denote the constant in this theorem when $s=1$ by $C_{n}$. The convergence

$$
\begin{aligned}
& \left(\Omega_{n} \bigcup \Omega_{n-1}\right) \backslash\left(\Omega_{n} \bigcap \Omega_{n-1}\right) \rightarrow \varnothing \\
& n \rightarrow \infty
\end{aligned}
$$

leads to $\underset{n \rightarrow \infty}{I} \rightarrow$, where $I$ is the identity operator.

From the proof of [9, Theorem 2] and the properties of maps $\Phi_{n}$ it follows that ${ }_{n \rightarrow \infty} \rightarrow I$. Since the sequence $\left\{C_{n}\right\}$ is bounded there exists an exact upper bound $\bar{C}=\max _{i=1,2 \ldots, n} C_{i}$. Satisfying the conditions of Theorem 1 we can assert that there exists a constant $C$ depending on the weak solution such that

$$
\|\| \circ \Phi_{n}-u_{n} \|_{n} \leq C(u) h_{n}^{k}, \quad n=0,1,2,3, \ldots .
$$

Using the recurrence formulae we obtain an optimal convergence rate for the full multigrid algorithm, which is the sense of the next theorem.

Theorem 2 Assume that the hypotheses H1-H5 hold, the number of iterations $p$ for solving the coarse-level residual problem fulfils $1<p \leq k^{2}-1$ and the number of iterations $r$ for executing the $n$-th level scheme satisfies $\gamma^{r} \rho<1$. Then

$$
\left\|u_{n}-\hat{u}_{n}\right\|_{n} \lesssim \delta h_{n}^{k}
$$

and

$$
\|\| u \circ \Phi_{n}-\hat{u}_{n} \|_{n} \lesssim(1+\delta) h_{n}^{k},
$$

where

$$
\delta=\gamma^{r} \frac{1+\rho}{1-\gamma^{r} \rho} \text { and } \rho=(k+\alpha)^{k} .
$$

Proof. The $n$-th level scheme (2) reduces the energy norm of the error in approximate solution by a factor of $\gamma$ per each iteration, i.e.

$$
\left\|u_{n}-\hat{u}_{n}\right\|_{n} \leq \gamma^{r}\left\|u_{n}-I_{n} \hat{u}_{n-1}\right\|_{n} .
$$

Obviously domI $I_{n}$ can be extended to the whole $H_{0}^{1}\left(\Omega_{n}\right)$ and then the results of [9, Theorem 2] will be true for all functions belonging to $H_{0}^{1}\left(\Omega_{n}\right)$.

Applying (4), Lemma 1, Theorem 1 and [9, Theorem 2] we get

$$
\begin{aligned}
& \left\|\mid u_{n}-\hat{u}_{n}\right\|_{n} \leq \gamma^{r}\left(\left\|u_{n}-u_{n} \circ \Phi_{n}\right\|_{n}+\left\|u_{n} \circ \Phi_{n}-I_{n} u_{n-1}\right\|_{n}\right. \\
& \left.+\left.||\left|I_{n} u_{n-1}-I_{n} \hat{u}_{n-1}\right|\right|_{n}\right) \\
& \leq \gamma^{r}\left(h_{n}^{k}+\left\|I_{n}\left(u_{n} \circ \Phi_{n-1}\right)-I_{n} u_{n-1}\right\|_{n}+\left\|\mid I_{n}\left(u_{n-1}-\hat{u}_{n-1}\right)\right\|_{n}\right) \\
& \leq \gamma^{r}\left(h_{n}^{k}+\left\|I_{n}\left(u_{n} \circ \Phi_{n-1}-u_{n-1}\right)\right\|_{n}+\left\|u_{n-1}-\hat{u}_{n-1}\right\|_{n-1}\right) \\
& \lesssim \gamma^{r}\left(h_{n}^{k}+\left\|\left|u_{n} \circ \Phi_{n-1}-u_{n-1}\right|\right\|_{n-1}+\left\|\mid u_{n-1}-\hat{u}_{n-1}\right\|_{n-1}\right) \\
& \lesssim \gamma^{r}\left(h_{n}^{k}+h_{n-1}^{k}+\left\||| u_{n-1}-\hat{u}_{n-1} \mid\right\|_{n-1}\right) \\
& \lesssim \gamma^{r}\left(h_{n}^{k}+\rho h_{n}^{k}+\|\| u_{n-1}-\hat{u}_{n-1} \|_{n-1}\right) \text {. }
\end{aligned}
$$

Thus we obtain

$\left\|u_{n}-\hat{u}_{n}\right\|_{n} \lesssim \gamma^{r}\left((1+\rho) h_{n}^{k}+\left\|\mid u_{n-1}-\hat{u}_{n-1}\right\|_{n-1}\right)$.

Apply (7) we estimate

$$
\begin{aligned}
& \|\| u_{n}-\hat{u}_{n} \|_{n} \lesssim \gamma^{r}\left[(1+\rho) h_{n}^{k}+\gamma^{r}\left((1+\rho) h_{n-1}^{k}+\left\|u_{n-2}-\hat{u}_{n-2}\right\|_{n-2}\right)\right] \\
& \leqslant \gamma^{r}\left[(1+\rho)\left(1+\gamma^{r} \rho\right) h_{n}^{k}+\gamma^{r} \mid\left\|u_{n-2}-\hat{u}_{n-2}\right\|_{n-2}\right] \\
& \leqslant \gamma^{r}\left[(1+\rho)\left(1+\gamma^{r} \rho\right) h_{n}^{k}+\gamma^{r}\left((1+\rho) \gamma^{r} h_{n-2}^{k}+\left\|u_{n-3}-\hat{u}_{n-3} \mid\right\|_{n-3}\right)\right] \\
& \leqslant \gamma^{r}\left[(1+\rho)\left(1+\gamma^{r} \rho+\left(\gamma^{r} \rho\right)^{2}\right) h_{n}^{k}+\left(\gamma^{r}\right)^{2}\left\|\mid u_{n-3}-\hat{u}_{n-3}\right\|_{n-3}\right] .
\end{aligned}
$$

Performing the recursion with respect to $n$ we complete the proof of (5) by

$\left\|u_{n}-\hat{u}_{n}\right\|_{n} \leqslant \gamma^{r}\left[(1+\rho)\left(1+\gamma^{r} \rho+\left(\gamma^{r} \rho\right)^{2}+\ldots+\left(\gamma^{r} \rho\right)^{n-1}\right) h_{n}^{k}\right.$ 


$$
\left.+\left(\gamma^{r}\right)^{n-1}\left\|u_{0}-\hat{u}_{0}\right\|_{0}\right] \leq \frac{(1+\rho) \gamma^{r} h_{n}^{k}}{1-\gamma^{r} \rho .}
$$

It is necessary to use the triangle inequality

$$
\left\|u \circ \Phi_{n}-\hat{u}_{n} \mid\right\|_{n} \leq\|\| u \circ \Phi_{n}-u_{n}\left\|_{n}+\right\| u_{n}-\hat{u}_{n} \|_{n}
$$

in order to obtain the validity of (6).

\section{NUMERICAL EXAMPLE}

Let $\Omega$ be a quarter of the unit disc. Consider a problem

$$
\left(\mathcal{P}_{\Delta}\right):\left\{\begin{array}{l}
\Delta u=\frac{2 x y}{x^{2}+y^{2}}, x \in \Omega, \\
u=0, x \in \Gamma
\end{array}\right.
$$

with the following exact solution

$$
u=\frac{x y \ln \left(x^{2}+y^{2}\right) .}{4}
$$

Let $\tau_{0}$ be an initial triangulation of the domain $\Omega$, Fig. (1) with four quadratic triangular finite elements. Consequently we obtain triangulations $\tau_{n}$, see Fig. (2) $n=1,2,3,4$ with $\left(2^{n+1}\right)^{2}$ elements and $2\left(2^{n+1}\right)^{2}-3.2^{n+1}+1$ internal nodes.

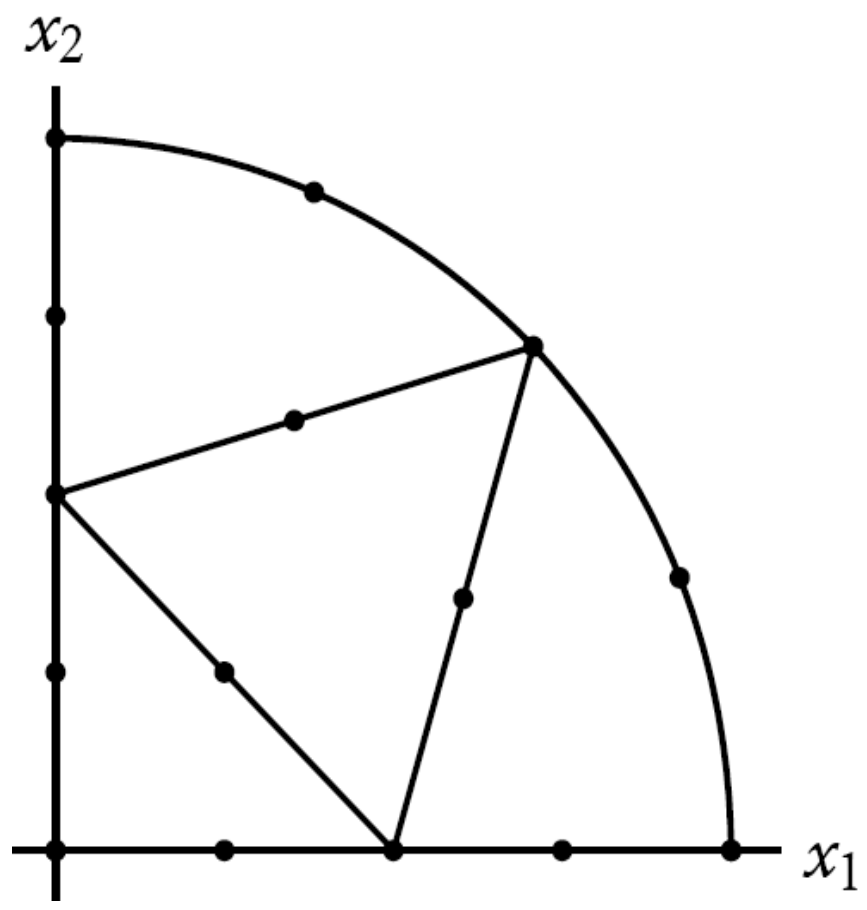

Fig. (1). An initial triangulation $\tau_{0}$ of the domain $\Omega$ by quadratic finite elements.

We can reduce the error in approximate solutions by increasing the number of presmoothing iterations $v$ or (and) by increasing the number of repetitions $r$ of the two-level scheme. It is very complicated to find optimal connection between the latter parameters with respect to the total computational work. Therefore we confine to the case $r=10, p=3$ and $v=20$. We follow the idea of Andreev,
Kascieva and Vanmaele [12] in order to obtain the approximate rate of convergence $\sigma$.

Having in mind that the equality (3) can be strengthened in view of

$$
k-\alpha<\frac{h_{n-1}}{h_{n}} \leq k, k \geq 2, \forall n \in \mathbf{N}
$$

for convex domains, we have

$$
\sigma \sim \frac{\ln \frac{\| \hat{u}_{n-1}-\left.I_{n-1} \hat{u}_{n-2}||\right|_{n-1}}{|| \hat{u}_{n}-I_{n} \hat{u}_{n-1}||_{n}}}{\ln 2}
$$

Table 1 confirms the theoreticl results obtained in Theorem 2 .

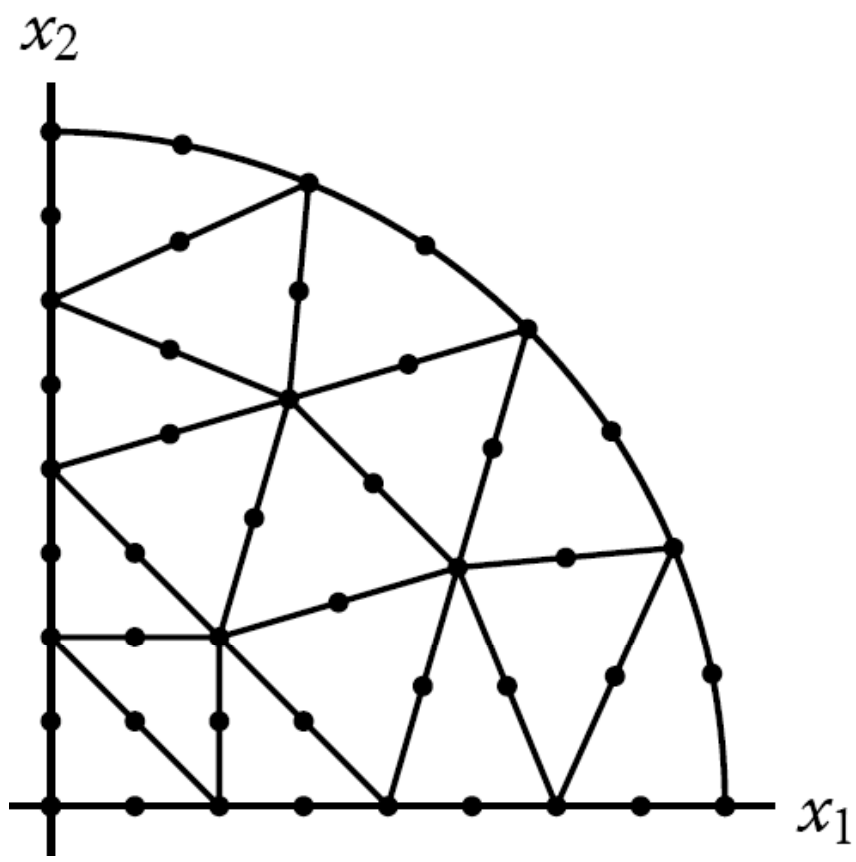

Fig. (2). A refined triangulation $\tau_{1}$ of the domain $\Omega$.

Table 1. Approximate Rate of Convergence Obtained by Three Diffeerent Meshes

\begin{tabular}{|c|c|c|c|}
\hline$\left\|\hat{u}_{3}-I_{3} \hat{u}_{2}\right\|_{3}$ & 0.0000238333 & $\left\|\hat{u}_{4}-I_{4} \hat{u}_{3}\right\|_{4}$ & 0.0000059082 \\
\hline$\left\|\hat{u}_{2}-I_{2} \hat{u}_{1}\right\|_{2}$ & 0.0001187984 & $\left\|\hat{u}_{3}-I_{3} \hat{u}_{2}\right\|_{3}$ & 0.0000238333 \\
\hline$\sigma$ & 2.31746 & $\sigma$ & 2.01219 \\
\hline
\end{tabular}

\section{CONCLUSION}

In this paper, we consider finite element discretizations of reaction-diffusion problems on two-dimensional curved domains with a Lipschitz-continuous boundary. The discretization is constructed by means of higher order elements on a sequence of nonnested triangulations. Optimal convergence rate is proved for the full nonnested multigrid algorithm. The convergence theorem is considered in the case when the number of iterations for the coarse-level residual problem is chosen such that the cost of the computational work of one iteration step of the multigrid algorithm is 
proportional to the number of unknowns. Numerical tests supporting the theory are presented.

\section{REFERENCES}

[1] Bramble JH, Pasciak JE, Xu J. The analysis of multigrid algorithms with nonnested spaces or noninherited quadratic forms. Math Comput 1991; 56: 1-34

[2] Neuss N, and Wieners C. Criteria for the approximation property for multigrid methods in nonnested spaces. Math Comput 2004; 73: 1583-1600.

[3] Scott R, Zhang S. Higher-dimensional nonnested multigrid methods. Math Comput 1992; 58: 457-66.

[4] Zhang S. An optimal order multigrid method for biharmonic, $C^{1}$ finite element equations. Numer Math 1989; 56: 613-24.

[5] Zhang S. Optimal-order nonnested multigrid methods for solving finite element equations I: on quasi-uniform meshes. Math Comput 1990; 55: 23-36.

[6] Zhang S. Optimal-order nonnested multigrid methods for solving finite element equations II: on non-quasi-uniform meshes. Math Comput 1990; 55: 439-50.
[7] Zhang S. Optimal-order nonnested multigrid methods for solving finite element equations III:on degenerate meshes. Math Comput 1995; 64: 23-49.

[8] Fidkowski KJ, Oliver TA, Lu J, Darmofal DL. p-Multigrid solution of high-order discontinuous Galerkin discretizations of the compressible Navier-Stokes equations. J Comput Phys 2005; 207: 92113.

[9] Jung M, Todorov TD. Isoparametric multigrid method for reactiondiffusion equations, Appl Numer Math 2006; 56: 1570-83.

[10] Lenoir M. Optimal isoparametric finite elements and error estimates for domains involving curved boundaries. SIAM J Numer Anal 1986; 23: 562-80.

[11] Ciarlet PG, Raviart PA. The combined effect of curved boundaries and numerical integration in isoparametric finite element method. Math. Foundation of the FEM with Applications to PDE. In: Aziz AK, Ed., New York: Academic Press 1972; pp 409-74.

[12] Andreev AB, Kascieva VA, Vanmaele M. Some results in lumped mass finite element approximation of eigenvalue problems using numerical quadrature formulas. J Comput Appl Math 1992; 43: 291-311.

(C) Todor D. Todorov; Licensee Bentham Open.

This is an open access article licensed under the terms of the Creative Commons Attribution Non-Commercial License (http://creativecommons.org/licenses/by-nc/3.0/) which permits unrestricted, non-commercial use, distribution and reproduction in any medium, provided the work is properly cited. 